\title{
NEAR GROUND AIR TEMPERATURE CALCULATION MODEL BASED ON HEAT TRANSFER OF VERTICAL TURBULENT AND HORIZONTAL AIR FLOW \\ Qinglin Meng, Lei Zhang*
}

State Key Laboratory of Subtropical Building Science South China University of Technology, Guangzhou 510640, China

Abstract: In order to calculate the air temperature of the near surface layer in urban environment, the surface layer air was divided into several layers in the vertical direction, and some energy balance equations were developed for each air layer, in which the heat exchange due to vertical turbulence and horizontal air flow was taken into account. Then, the vertical temperature distribution of the surface layer air was obtained through the coupled calculation using the energy balance equations of underlying surfaces and building walls. Moreover, the measured air temperatures in a small area (with a horizontal scale of less than $500 \mathrm{~m}$ ) and a large area (with a horizontal scale of more than $1000 \mathrm{~m}$ ) in Guangzhou in summer were used to validate the proposed model. The calculated results accord well with the measured ones, with a maximum relative error of $4.18 \%$. It is thus concluded that the proposed model is a high-accuracy method to theoretically analyze the urban heat island and the thermal environment.

Keywords: turbulent heat exchange; urban thermal environment; near surface layer; air temperature; calculation model

\section{INTRODUCTION}

The surface layer is a system of the atmospheric boundary layer close to the ground with the thickness being $1 / 10$ of the whole atmospheric boundary layer. Generally, it is considered that the thickness of surface layer is several hundred meters. The human being lives within the surface layer mainly, so it keeps a close relationship with human being's life. At present, the issue of surface layer over special underlaying surface such as surface layer of urban areas and different vegetations as well as the mutual relationship between it and underlaying surface is still researchable.

\section{SURFACE THERMAL EQUILIBRIUM QUATION}

The thermal equilibrium of any surface $i$ in the area can be expressed as Formula (1), where these surfaces include roof, external wall and ground.

$$
S \downarrow_{i}-S \uparrow_{i}+L \downarrow_{i}-L \uparrow_{i}=Q_{c(i)}+Q_{d(i)}+Q_{e(i)}
$$

Where $S$ is short wave radiation, $L$ is long wave radiation, $\downarrow \uparrow$ are for downward and upward radiation, Qc is convection heat exchange between air and surface, $Q d$ is conduction heat transfer of solid meterial, $Q e$ is evaporation heat transfer.

\section{THERMAL EQUILIBRIUM OF SURFACE LAYER AIR}

The thermal transfers of air and external environment are:

(1) Sensible thermal transfer between underlaying surface and surface layer;

(2) Energy brought and taken away through air inflow and outflow;

(3) Sensible thermal transfer between external building wall and ambient air;

(4) Anthropogenic Heat.

The thermal transfer within the surface layer includes mainly: thermal transfer caused by air molecular diffusion and turbulence. As for the thermal equilibrium schematic of surface layer air, see Fig. 1.

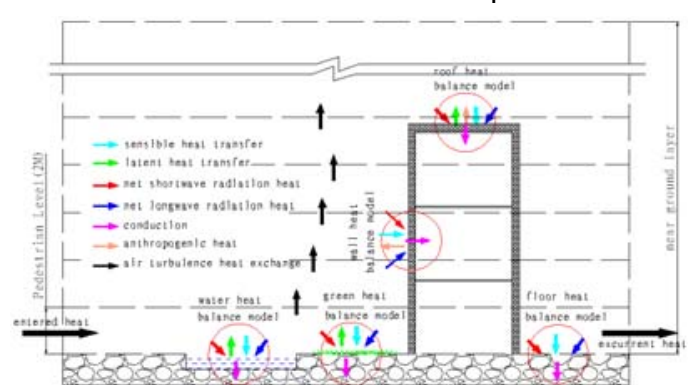

Fig.1 Near ground air heat balance model In the urban surface layer shown in Fig. 1, take out a thin layer along with the contour, then the thermal equilibriums considered are:

(1) Horizontal air flow: if the mass flow in the area within unit time is $V_{m i}$, the heat brought is $C_{a} V_{m i} T_{\text {in }}$ and if the mass flow in the area is $V_{m o}$, the heat taken away is $C_{a} V_{\text {mo }} T$, the internal heat change in the thin layer caused by horizontal air flow within unit time can be obtained from Formula (2).

$$
Q_{1}=C_{a} V_{m i} T_{i n}-C_{a} V_{m o} T
$$

Where $C_{a}$ is air specific heat, $T_{i n}$ is incoming air temperature, $T$ is mean air temperature of the thin layer.

(2) Internal thermal source: the internal thermal source in the thin layer is divided into two parts, Part 1 is the sensible thermal transfer between other surface (wall surface) and air of the thin layer; Part 2 is the man-made heat dissipating capacity of other surface in the thin layer.

$$
Q_{2}=\left[\sum_{i=1}^{n} H_{c, i} A_{i}\left(T_{s, i}-T\right)+\sum_{i=1}^{n} Q_{a, i} A_{i}\right]
$$

Where $H_{c, i}$ is convection heat exchange between air and surface i, $A_{i}$ is area of surface i, $T_{s, i}$ is external surface temperature of surface i, $Q_{a, i}$ is anthropogenic heat of unit area. 
(3) Diffusion: the heat from surface $z$ to the thin layer within unit time due to diffusion is $-C_{a} \rho A\left(D+K_{t}\right) \frac{\partial T}{\partial z}$, the heat from Surface $z+\Delta z$ to the thin layer is $-C_{a} \rho A\left(D+K_{t}\right) \frac{\partial T}{\partial z}+\frac{\partial}{\partial z}\left[-C_{a} \rho A\left(D+K_{t}\right) \frac{\partial T}{\partial z}\right] d z$, the heat change caused within unit time due to diffusion can be obtained from Formula (4):

$Q_{3}=\frac{\partial}{\partial z}\left[C_{a} \rho A\left(D+K_{t}\right) \frac{\partial T}{\partial z}\right] d z$

Where $D$ is air molecular thermal diffusion coefficient, $K_{t}$ is air turbulence thermal diffusion coefficient, $A$ is horizontal area exclude building area, $d z$ is thickness of thin layer.

The internal heat change of the above thin layer that will cause the air temperature change in the thin layer can be obtained from Formula (5):

$Q_{4}=-C_{a} \rho \frac{\partial T}{\partial \tau} A d z$

Formula (6) can be obtained from the thermal equilibrium in the thin layer.

$Q_{1}+Q_{2}+Q_{3}+Q_{4}=0$

Put the above expressions of $Q_{1} \sim Q_{4}$ into Formula (6), Formula (7) can be obtained after the arrangement.

$M_{a, j} C_{a} \frac{\partial T}{\partial \tau}=\left(C_{a} V_{m i} T_{i n}-C_{a} V_{m o} T\right)+C_{a} \rho A\left(D+K_{t}\right) d z \frac{\partial^{2} T}{\partial z^{2}}+Q_{v}$

Where $M_{a, j}$ is weight of air in the thin layer, $Q_{v}$ is inner heat source.

The Equation (7) is the control equation of the air temperature model of surface layer. After the discreteness if this equation, if the initial condition and boundary condition are given, all air temperatures can be obtained through forward elimination and backward substitution.

\section{CALCULATION OF HEAT EXCHANGE COEFFICIENT OF TURBULENCE}

According to the first order closure scheme ( $\mathrm{K}$ theory), the air turbulent motion is similar to the air molecular motion. And the turbulence thermal transmission is similar to molecular thermal transmission, so the thermal flux caused by turbulence can be:

$H_{t}=-c \rho K_{t} \frac{d t}{d z}$

When the aerosphere section is unstable and the turbulence transmission is much faster than molecular transmission, $K_{t} \gg D$, and at this time, the thermal transmission in the air $H=H_{d}+H_{t} \approx H_{t}$, so due to unit mass temperature change caused by thermal transmission in the air can be expressed as:

$\frac{d T}{d \tau}=K_{t} \frac{\partial^{2} T}{\partial z^{2}}$

That is to say the turbulence thermal diffusivity is subject to typical heat conduction equation, where $K_{t}$ value changes in accordance with the change of air condition.

The turbulence thermal diffusivity is not the fixed air physical property parameter but a changeable parameter at any time, so it can only be obtained through iterative computation based on area roughness, air temperature, air velocity and stableness. $K_{t}$ can be expressed as Formula 10, see references (Zhang Qiang, Lv Shi-hua, 2003; Gao Zhiqiu, et al., 2002; Jun Tanimoto, et,al., 2004; Sang-Hyun Lee, Soon-Ung Park, 2008; Ian N. Harman, John J. Finnigan, 2007; David Hamlyn, et,al., 2007) for concrete calculation methods.

$K_{t}=\kappa u_{*} z / \phi_{2}$

Where $\kappa$ is karman constant, $u_{*}$ is friction velocity, $z$ is vertical height, $\phi_{2}$ is profile function of air temperature.

\section{MODEL VERIFICATION AND RESULT}

In order to verify the accuracy of the above theoretical model and calculation method, the measurement results for the small sized area (Donghu area of the South China University of Technology) and large sized area (Guangzhou University City) in Guangzhou are adopted.

The air temperature of Donghu area of the South China University of Technology was measured from July 9 to 132007.

The meteorological data recorded in weather station during the measurement was adopted as the incoming flow parameter. And the average air temperature within the human activities height $(2 \mathrm{~m})$ obtained from the calculation model for surface layer air temperature mentioned in this text was adopted and compared with the measured result 
of Donghu area, see Fig. 2 and Table 1.

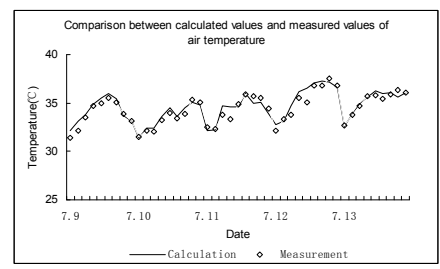

Fig.2 Comparison between calculated values and measured values

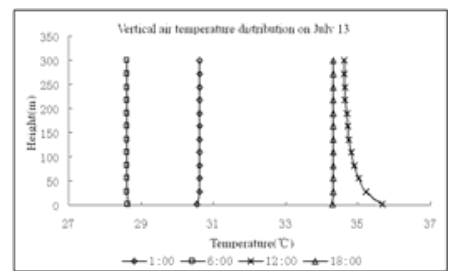

Fig.3 Vertical air temperature distribution of East Lake

Table1 Statistics analysis of calculated values and measured values of air temperature at East Lake area

\begin{tabular}{cccc}
\hline Item & Sample & Mean & Standard Deviation \\
\hline Measurement $/ \square$ & 45 & 34.38 & 1.57 \\
Calculation/ $\square$ & 45 & 34.59 & 1.54 \\
absolute error $/ \square$ & 45 & 0.43 & 0.33 \\
Relative error $/ \%$ & 45 & 1.25 & 0.98 \\
\hline
\end{tabular}

From Fig. 2 and Table 1, the change trends of calculated values and measured values are similar to each other. The maximum relative error is $4.18 \%$, which was occurred at $13: 00$ on July 12 . The average relative error is $1.25 \%$, and number of hours with relative errors being less than $3 \%$ accounts for $93.3 \%$ of those for measurement, from which it can be concluded that the accuracy of average temperature obtained through this model for human activities height is much higher.

The error between measured air temperature and calculated value is caused probably by the following points:

(1) Some simplified and assumed treatments of model caused the error, for instance, other surfaces were probably under insulation boundary conditions in addition to the heat exchange along with the air flow, which would reduce the heat exchange between the calculated area and other areas in parallel with the air flow;(2) The complicated geometrical relation of outdoor environment caused the error. Some simplified treatments were performed for the model when the angle factor and shadow factor were calculated, causing the main heat source of the area; deviation occurred during calculation of solar radiation;(3) During this calculation, influence of man-made heat was not considered, which was one of the reasons for causing errors.

The air temperature vertical distribution diagram for Donghu area at four typical hours on July 13 was made as Fig. 3.

From Fig. 3, the temperature of surface layer is under a temperature inversion condition at 1:00 am, where air temperature the bottom is $30.54{ }^{\circ} \mathrm{C}$, while that of the top is $30.61{ }^{\circ} \mathrm{C}$. And at $6: 00$ during sunrise and $18: 00$ during sunset, the temperature gradient is low, which is isothermal. At $12: 00$ at noon, the bottom is $35.66{ }^{\circ} \mathrm{C}$, while that of the top is $34.62^{\circ} \mathrm{C}$, showing that the temperature of surface layer is unstable. In addition, during the calculation for the temperature of surface layer for Donghu area, the calculated surface layer height is $300 \mathrm{~m}$, showing that at 12:00 at noon, the influence of the thermal environment of underlaying surface on the temperature of surface layer can reach up to about $300 \mathrm{~m}$; when this height is exceeded, the surface layer height calculated is added, the influence on the air $2 \mathrm{~m}$ away from the ground will be less than $0.1^{\circ} \mathrm{C}$. According to Document: the thickness of surface layer will change along with the change of stability; when it is unstable, it can reach up to $100 \mathrm{~m}$ or even higher; when it is stable, it can be only $10 \mathrm{~m}$ or $20 \mathrm{~m}$. The analysis result of this text is similar to the description of this document, showing that the calculation model mentioned in the text is reasonable for the description of the air temperature of surface layer.

The comparison between the air temperatures of Guangzhou University City measured from August $28 \sim 30$ 2007 and those calculated from the model is adopted.

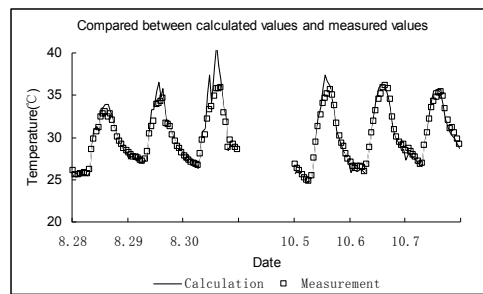

Fig.4 Compared between calculated value and measured value

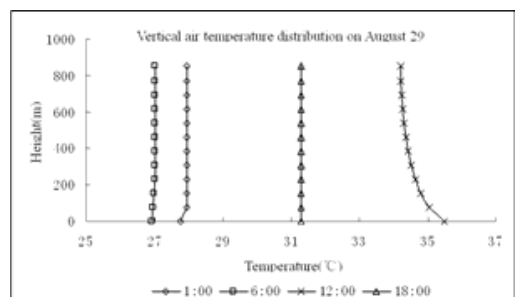

Fig.5 Vertical air temperature distribution of Guangzhou University City

Table2 Statistics of calculated value and measured value

\begin{tabular}{cccc}
\hline Item & Sample & Mean & Standard Deviation \\
\hline Measurement/ $\square$ & 144 & 30.06 & 3.11 \\
Calculation/ $\square$ & 144 & 30.25 & 3.68 \\
Absolute error $/ \square$ & 144 & 1.96 & 2.26 \\
Relative error $/ \%$ & 144 & 0.63 & 0.78 \\
\hline
\end{tabular}

During the two comparing periods, the average relative error is $1.96 \%$, where the hours with relative error being 
less than $5 \%$ account for $93.7 \%$ of the whole period; the hours with relative error being more than $5 \%$ account for $6.3 \%$ of the whole period; the hours under day time solar radiation with higher temperature is 10:00 15:00; the hours with relative error being less than $1 \%$ account for $41 \%$ which are almost under night. This can show further that the underlaying surface is the main heat resource for surface layer air. During day time when the influence of solar radiation on underlaying surface temperature is high, the calculation for underlaying surface temperature is determined by the calculation for air temperature.

The temperature variation at vertical direction of Guangzhou University City area at four typical hours on August 29 was made too, see Fig. 5. It is shown that the temperature variation trend at the height direction of Guangzhou University City area at four typical hours is similar to that of Donghu area, where the difference is that, due to the large size of the university area, the influence of underlaying surface on the air temperature of surface layer rises, at noon of August 29, the maximum influenced height can reach up to $850 \mathrm{~m}$.

\section{CONCLUSION}

In this article, the surface layer of urban area at vertical direction is divided into several sub-layers, and energy balance equations for all air sub-layers are established, where the heat exchange of turbulence at vertical direction and that of incoming air at horizontal direction are considered. From the combined calculation of ground and building wall surface equations, the air temperature of surface layer is distributed vertically.

From the comparison between the measured air temperature of small sized Donghu area and average air temperature calculated through this model within $2 \mathrm{~m}$ high from the ground, the error from both area are low, the maximum relative error is $4.18 \%$ and the average relative error is $1.25 \%$.

From the comparison between the results measured from August 28 30 and October $5 \sim 72007$ for Guangzhou University City, the average relative error for the measured value and calculated value is $1.96 \%$, hours with relative error being less than $5 \%$ account for $93.7 \%$ of the whole period.

The calculation model for air temperature of surface layer mentioned in this text can meet the accuracy requirement of thermal environment research basically, and can be used for the calculation for air temperature of surface layer of urban area and is suitable for the simplified formula for WBGT as well as predicting thermal environment quality after performance of urban plan and building design plan.

\section{ACKNOWLEDGEMENTS}

The study was supported by National Natural Science Foundation of China (Project No. 50538040, 50720165805, 50808083), Natural Science Foundation of Guangdong Province of China (Project No. 8251064101000006), National Key Technology R\&D Program of China (Project No. 2006BAJ02A05-05), the 111 Project (Project No. 111-2-13) and the project funded by the State Key Laboratory of Subtropical Building Science, South China University of Technology, China (Project No. 2008ZB14).

\section{REFERENCES}

David Hamlyn, Trevor Hilderman, Rex Britter. A simple network approach to modelling dispersion among large groups of obstacles [J]. Atmospheric Environment, 2007,41(28): 5848-5862.

G. Mills. An Urban Canopy-Layer Climate Model [J]. Theoretical and Applied Climatology. 1997.57(3-4):229-244.

Gao Zhiqiu, Bian Lingen, Lu Changgui, et al.Estimation of aerodynamic parameters in urban areas [J]. Quarterly Journal of Applied Meteorlolgy, 2002,13(1):26-33.

Hiroyuki Kusaka, Hiroaki Kondo, Yokihiro Kikegawa, et al. A Simple Single-Layer Urban Canopy Model For Atmospheric Models: Comparison With Multi-Layer And Slab Models [J]. Boundary-Layer Meteorology, 2001,101(12): 329-358.

Hiroaki Kondo, Yutaka Genchi, Yukihiro Kikegawa, et al. Development of a Multi-Layer Urban Canopy Model for the Analysis of Energy Consumption in a Big City: Structure of the Urban Canopy Model and its Basic Performance [J]. Boundary-Layer Meteorology, 2005,116(9): 395-421.

lan N. Harman, John J. Finnigan. A simple unified theory for flow in the canopy and roughness sublayer [J]. Boundary-Layer Meteorol, 2007,123(5):339-363.

Jun Tanimoto, Aya Hagishima, Parichart Chimklai. An approach for coupled simulation of building thermal effects and urban climatology [J]. Energy and Buildings, 2004.36: 781-793.

Meng Qinglin, Chen Qigao, Ran Maoyu,et al. Derivation of the vaporising heat transfer coefficient he [J]. Acta Energiae Solaris Sinica, 1999, 20(2):216-219.

Ran Maoyu, Yang Ruohan, Meng Qinglin. Prediction model and calculation method of the boundary surface temperature of out door space under solar radiation [J]. Acta Energiae Solaris Sinica, 2006,27(7):719-724.

Sang-Hyun Lee, Soon-Ung Park. A Vegetated Urban Canopy Model for Meteorological and Environmental Modelling [J]. Boundary-Layer Meteorol, 2008,126(1): 73-102.

Xiao Yongquan, Wang Fei. Building envelope's dynamic heat balance model under solar radiation and example analysis [J]. Acta Energiae Solaris Sinica, 2006, 27(3):270-273.

Zhang lei, Meng Qinglin, Shu Lifan. Analysis on Dynamic Thermal Balance Model of Landscape Water and its Numerical Simulation [J]. Building Science, 2007,23(10):58-61.

Zhang Qiang, Lv Shi-hua. The Determination of Roughness Length over City Surface [J]. Plateau Meteorology, 2003,22(1):24-32. 